\title{
Models and Algorithms for the Optimization of Real-World Routing and Logistics Problems
}

\author{
Stefano Novellani
}

Received:

This is a summary of the author's Ph.D. thesis supervised by Daniele Vigo (Università di Bologna) and Manuel Iori (Università di Modena e Reggio Emilia) and defended on 10 April of 2015 at the Department of Electrical, Electronic, and Information Engineering - "Guglielmo Marconi” (DEI), Università di Bologna, Italy. The thesis is available from the author upon request at stefano.novellani@ unimore.it.

Logistics and transportation aspects are very important and represent a relevant cost for producing and shipping companies, but also for public administration and private citizens. The optimization of resources and the improvement in the organization of operations is crucial for all branches of logistics, from the operation management to the transportation. Optimization techniques, models, and algorithms represent important methods to solve the always new and more complex problems arising in different segments of logistics. Many operation management and transportation problems are related to the optimization class of problems called Vehicle Routing Problems (VRPs). In the thesis we considered several real-world deterministic and stochastic problems that are included in the wide class of the VRPs, and we solved them by means of exact, heuristic, and metaheuristic methods. In particular, we treated three classes of real-world routing and logistics problems.

In the first part of the thesis we dealt with one of the most important tactical problem that arises when managing bike sharing systems, that is the Bike sharing Rebalancing Problem (BRP), a multi vehicle many-to-many one-commodity pickup and delivery VRP. We formally defined the BRP, for which we supplied a set of formulations and good inequalities included in well performing branch-and-cut algorithms. We tested the algorithms on newly collected real-life instances. This work resulted into a publication: "The bike sharing rebalancing problem: Mathematical formulations and benchmark instances”, M. Dell'Amico, E. Hadjicostantinou, M. Iori, S. Novellani. Omega 45, 7-19 (2014).

Furthermore, we tackled the BRP with a destroy and repair algorithm including novel properties to reduce the complexity of the components of the algorithm. We also solved the generalization of the BRP where maximum duration constraints for the routes are considered, the so-called one-commodity Pickup and Delivery VRP (1- 
PDVRP), for which we proposed new inequalities included in a branch-and-cut framework. We conjointly showed the adaptation of the destroy and repair algorithm to solve the 1-PDVRP. We gained optimality for all the small instances and significantly improved upon the literature results. This work has been accepted for publication as "A destroy and repair algorithm for the Bike sharing Rebalancing Problem" M. Dell'Amico, M. Iori, S. Novellani, T. Stützle. Comput Oper Res 71, 149-162 (2016).

Eventually, we defined the Stochastic BRP (SBRP), the version of the BRP with stochastic demands. We provided different models for the SBRP and solved them with L-Shaped methods and branch-and-cut algorithms. Moreover, we presented heuristic algorithms for the SBRP that account for the negative and positive correlations between the nodes in the solution construction. We obtained good results on real-world instances.

In the second part of the thesis, we proposed models and algorithms for real-world earthwork optimization problems. We presented a novel two-phase earthwork optimization model for highway construction, considering many real-world features for the first time. Moreover, we presented a Decision Support System (DSS) for highway construction developed for the Autostrada Pedemontana Lombarda highway project in collaboration with one of the major construction companies in Europe. The DSS includes easy-to-use graphical and optimization components and it is now applied to several highway construction projects. This work converted into two papers published on international journals.

In the last part of the thesis, we considered the optimization of the threeDimensional Printing (3DP) process. By describing it, we highlighted several optimization issues regarding the 3DP. Among those, we described the problem related to the tool path definition in the 3DP process that we called the 3D Routing Problem (3DRP), a generalization of the well-known arc routing problem. We presented an ILP model and several heuristic algorithms to solve the 3DRP obtaining good results on real-world instances with respect to results of freeware available on the Internet.

Overall, the Ph.D. thesis considered many real-world new problems, and solved them with novel algorithms providing good results. 